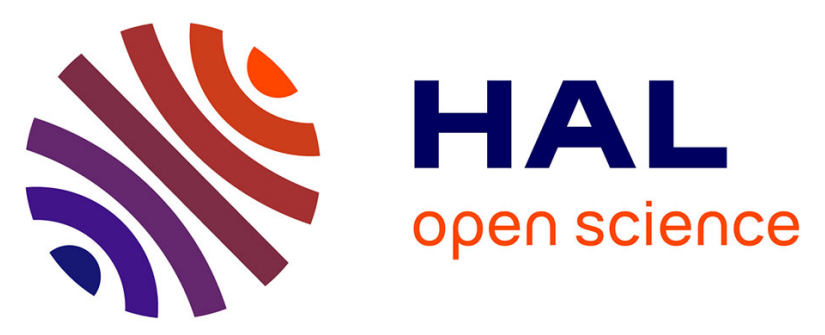

\title{
Impact of cigarette smoke on physical-chemical and molecular proprieties of human skin in an ex vivo model
}

Giuseppe Percoco, Angela Patatian, Florine Eudier, Michel Grisel, Thomas

Bader, Elian Lati, Géraldine Savary, Celine Picard, Philippe Benech

\section{- To cite this version:}

Giuseppe Percoco, Angela Patatian, Florine Eudier, Michel Grisel, Thomas Bader, et al.. Impact of cigarette smoke on physical-chemical and molecular proprieties of human skin in an ex vivo model. Experimental Dermatology, 2021, 30 (11), pp.1610-1618. 10.1111/exd.14192 . hal-02948022

\section{HAL Id: hal-02948022 \\ https://hal.science/hal-02948022}

Submitted on 25 Sep 2020

HAL is a multi-disciplinary open access archive for the deposit and dissemination of scientific research documents, whether they are published or not. The documents may come from teaching and research institutions in France or abroad, or from public or private research centers.
L'archive ouverte pluridisciplinaire HAL, est destinée au dépôt et à la diffusion de documents scientifiques de niveau recherche, publiés ou non, émanant des établissements d'enseignement et de recherche français ou étrangers, des laboratoires publics ou privés. 
4

\title{
Impact of cigarette smoke on physical-chemical and molecular proprieties of human skin in an ex vivo model
}

\author{
PERCOCO Giuseppe ${ }^{1 *}$, PATATIAN Angela ${ }^{2 * \#}$, EUDIER Florine $^{3}$, GRISEL Michel ${ }^{3}$, \\ BADER Thomas $^{2}$, LATI Elian ${ }^{1,2}$, SAVARY Géraldine ${ }^{3}$, PICARD Céline ${ }^{3 \#}$, BENECH \\ Philippe ${ }^{2,4}$
}

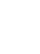

1, BIO-EC Laboratory, 1 chemin de Saulxier 91160 Longjumeau, France

2, Genex Laboratory, 1 chemin de Saulxier 91160 Longjumeau, France

3, Normandie Univ, France; UNILEHAVRE, FR 3038 CNRS, URCOM, EA 3221, 25 rue Philippe Lebon BP 1123, 76063 Le Havre cedex, France

4, Aix Marseille Université, CNRS, INP, Inst Neurophysiopathol, Marseille, France.

*: These authors have contributed equally to the present work

\#: Corresponding authors:

Celine PICARD: celine.picard@univ-lehavre.fr

Angela PATATIAN: a.patatian@ laboratoire-genex.fr 
ABSTRACT

Background: This is a study about the skin aging exposome, focusing on the effect of cigarette smoke. Human living skin explants (HSE) were exposed to cigarette smoke (CS) of two cigarettes for 2 hours using a custom-made exposure chamber, the Pollubox ${ }^{\circledR}$. Effects on the surface physico-chemistry and molecular properties of the skin were analyzed and reported for the first time. Basic Procedures: To this end, transcriptomic study followed by immunohistochemistry, malondialdehyde dosage (MDA) and surface physio-chemistry data (surface free energy determination, TEWL, skin pH and FTIR spectroscopy of the explant) were collected from non-treated and treated HSE.

Main Findings: Results showed a decrease of the total surface free energy of the treated HSE. This decrease reflected higher interactions with polar compounds from the environment and consequently a decrease of the surface hydrophobicity. Additionally, an increase of TEWL and skin pH was observed after treatment. The transcriptomic analysis showed downregulation of mitochondrial genes (PON2-NDUFA4L2ATP1A1-ALDH2-PRODH) combined with an increase of MDA in CS-treated HSE. Conclusions: CS-induced oxidation of lipids at HSE surface alters the skin barrier: interactions with polar products are enhanced and the lipid chain packing at the surface is modified. Consequently, skin permeability could increase which correlated with repression of CA9 and AQP1 genes. Beside activation of AHR-NRF2 pathway in CS-exposed HSE, our results suggested that mitochondrial functions were strongly impacted and oxidized lipids failed to be eliminated promoting skin barrier alteration. A mitophagy activity was suggested through the confirmation of PINK1 accumulation in the epidermis by immunostaining.

\section{KEYWORDS:} cigarette smoke-skin exposome -mitochondrial alterations -surface physico chemistry-transcptomic analysis 


\section{INTRODUCTION}

The exposome includes the totality of external factors to which human beings are exposed during their life [1]. The concept of the skin aging exposome has been defined by Krutman and collaborators [2] as the whole external and internal factors to which humans are exposed daily and the response of the human body to these factors promoting an accelerated skin aging process.

It has been proposed that the skin aging exposome is composed of seven different categories, including sun radiation, tobacco, temperature, nutrition, stress, lack of sleep and air pollution. Environmental cigarette smoke (CS) is one of the major harmful components of the human skin exposome.

Most commonly called secondhand smoke, it is a complex and reactive mixture which contains over 5000 chemicals including hazardous amines, carbonyls, hydrocarbons and metals among others emitted from the burning tobacco made up of the mainstream smoke exhaled by smokers and sidestream smoke diluted with ambient air [3].

Many studies were concerned with the detection and characterization of the hazardous components in mainstream smoke $[4,5]$, and their effects on human health have been largely documented in response to active smoking [6].

Additionally, environmental CS exposure can cause several illnesses in non-smokers including ischaemic heart diseases in adults, lower respiratory infections, and asthma in adults and children, among other adverse health effects [7,8,9].

Furthermore, the macroscopic effects of CS on skin are largely described and recognized. From the beginning of the seventies it has been demonstrated that wrinkle formation is a main clinical symptom related to tobacco consumption [10] and ever since the visible impact of tobacco smoke on human skin has been reported by different studies in vitro and in vivo [11, 12]. However, the molecular mechanisms impacted by CS in cutaneous tissue have been investigated only recently.

For the first time in 2001, Lahmann and collaborators [13] have indicated that the skin of smokers expresses higher levels of matrix metalloproteinase 1 (MMP1) compared to nonsmokers. The negative effects of CS on the extracellular matrix (ECM) were confirmed by studies in mice showing that the skin exposed to CS extracts presents a decreased level of collagen staining [14] and exhibited an alteration of collagen biosynthesis. In parallel, it has been shown that CS can increase reactive oxygen species (ROS) formation in different cell 
types, including fibroblasts [15]. ROS are responsible for the induced expression of different MMPs and activity resulting in degraded extracellular matrix and accelerated skin aging. Most recently, the implication of the aryl hydrocarbon receptor (AhR) in skin responses to CS have been also established in vitro [16]. In particular, it has been shown that upon exposure to tobacco extracts, keratinocytes express higher levels of MMP-1 and CYP1B1 in an AhRdependent manner. Likewise, the role of AhR in CS-induced skin hyperpigmentation was also investigated [17].

Moreover, the relationship between smoking and the development of several cutaneous skin diseases, including atopic dermatitis [18], psoriasis [19] and impaired wound healing [20] have been studied.

Given the importance and the necessity to characterize in more detail the impact of CS on the different organs in humans, including skin, several experimental systems have been developed for the exposure and the delivery of tobacco smoke in vitro [21]. Indeed, Lecas and collaborators [22] have implemented a reconstructed epidermis-based model in order to investigate the impact of tobacco smoke on the epidermis. This study has shown that CS exposure triggers different events in human epidermis including an oxidative stress evaluated by 4-hydroxynonenal immunostaining, an overexpression of ECM degrading enzymes such as MMP-1 and MMP-3, and an increase of the pro-inflammatory interleukins, IL1 alpha, IL-8 and IL-18. Interestingly, they observed also a repression of a group of genes encoding proteins involved in skin barrier functions, cell-cell junctions and cell adherence.

The surface physico-chemistry is an important parameter to determine skin interactions with its surrounding environment [23]. Mavon et al. [24] have demonstrated that in-vivo human skin surface is a hydrophobic monopolar basic surface. Thus, it may strongly interact with bipolar substances and with monopolar ones with opposite sign. This behavior depends on the specific topography and lipid composition (stratum corneum) of the area of the body. Despite this interest, so far, no studies investigated the impact of CS on this parameter of human skin. Interestingly, Eudier et al. [23] have recently highlighted that HSE behaved in a very similar way as in vivo skin in terms of surface physico-chemistry and are a precious alternative to study skin surface interactions with urban pollutants or CS.

Thus, our aim was to study the effects of environmental cigarette smoke on the physicochemical properties of the stratum corneum (SC). To that purpose, HSE were exposed to CS using the exposure chamber Pollubox ${ }^{\circledR}$ which reproduces a condition of passive smoking. The physico-chemical properties of the surface of the HSE were measured before and after exposure. For the first time the surface free energy parameters of non-treated and treated HSE 
114 are documented. The parameters relevant to the skin barrier function modifications included 115 measurements of trans-epidermal water loss, skin $\mathrm{pH}$ and the lipid chain packing in the 116 stratum corneum. Additionally, the impact of CS on the human skin transcriptome was 117 investigated.

118 


\section{Materials and methods}

\section{Preparation of human skin explants}

122 Human living skin explants were obtained from plastic surgery of the abdominal area of six

123 different donors (Table S1), in accordance with the Declaration of Helsinki and after the

124 patients had given informed consent. HSE have been prepared as described by Percoco et al.

1252013 [25]. For surface characterization square HSE (2cm x $2 \mathrm{~cm})$ have been used.

\section{Exposure of human skin explants to cigarette smoke}

In order, to define whether exposure of HSE with 1 or 2 cigarettes can induce an efficient stress activation in HSE the expression of AHR, NRF2 target genes and expression of MMP1/MMP3 as well as pro-inflammatory genes, IL1b and IL6 were evaluated first (Table S2). The following results were obtained after 5 days of culture with HSE, exposed to smoke from 2 cigarettes of a US brand for 2 hours using the exposure chamber PolluBox®. For microarrays, immunostaining and malondialdehyde (MDA) dosage, HSE were sampled 24hrs after CS exposure (See supplement data, Materials and methods). Explants from three different donors have been analyzed (Table S1) and for each condition (control and CSexposed sample) 3 explants were used, except for MDA dosage where 4 samples per condition were analyzed.

138 For surface characterization after CS exposure, HSE were mounted on a specific support in order to stretch the explant surface and subsequently sent to URCOM laboratory for further analysis after 48 hours following CS exposure. For each condition 5 explants were used 


\section{Contact angle measurements and surface free energy determination}

145 Skin surface physico-chemistry is an important means to evaluate interactions of the surface 146 of the skin, our first barrier, with its environment and, as a consequence, skin permeability 147 [24]. As detailed above, the Pollubox ${ }^{\circledR)}$ allows nebulization of CS similar to a real skin exposure. Contact angle measurements were performed $48 \mathrm{~h}$ after previous exposure to CS (2h) or on control explants, using three reference liquids (water, diiodomethane, and formamide). The values of these angles are reported in figure 1. Contact angle measurements characterize the wettability of a surface by a liquid which depends on the intermolecular interaction between the two. Thus, the smaller the angle, the higher the liquid spreads, indicating a better intermolecular interaction.

According to ANOVA performed on recorded data (Fig. 1a), CS treatment has a significant impact on explant physico-chemistry. Indeed, the water contact angle decreased by more than $15^{\circ}$ after CS treatment $(\mathrm{p}<0.0001)$ indicating a decreased hydrophobicity; while the diiodomethane contact angle increased by $8^{\circ}(\mathrm{p}<0.0001)$. The formamide contact angle slightly decreased not significantly. Those changes indicated that upon CS treatment, interactions with polar liquids such as water were reinforced and interactions with apolar liquid such as diiodomethane were weakened. This was a first indication that CS treatment modified skin polarity.

The Van Oss model was used to determine the surface free energy (SFE) [26,27]. This model allows splitting SFE into two components: the Lifshitz-van der Waals component $\left(\gamma^{\mathrm{LW}}\right)$, which represents the van der Waals (apolar) interactions (Keesom, Debye, London), and the acid-base component $\left(\gamma^{\mathrm{AB}}\right)$, representing the acidic and basic interactions, according to the Lewis concept. In this model, acid-base component is composed of two parameters: $\gamma^{+}$ (electron acceptor, Lewis acid) and $\gamma^{-}$(electron donor, Lewis base). $\gamma^{\mathrm{AB}}$ component includes the hydrogen bounding and the $\pi$ - electron interactions.

Surface free energy parameters calculated from those measurements also showed a significant impact of CS treatment (Fig. 1b). $\gamma^{\mathrm{LW}}$ component significantly decreased by $6 \mathrm{~mJ} / \mathrm{m}^{2}$. This means that the dispersive interactions of CS-treated explants appear weaker than of control 
explants. In parallel, the basic component $\left(\gamma^{-}\right)$was increased by more than $10 \mathrm{~mJ} / \mathrm{m}^{2}$. Nevertheless, as the acidic component $(\gamma+)$ remained close to zero, the increase of $\gamma^{-}$did not significantly impact the $\gamma^{\mathrm{AB}}$ value $(\mathrm{p}>0.05)$. On the other hand, the increase in the electron donor component means that the monopolar basic behavior of skin surface was reinforced with an increase of the electron density at the extreme surface. This specific behavior was already evidenced by Eudier et al. [28] dealing with squalene oxidation by urban pollutants. The described surface free energy parameters modifications induced a significant lowering of the total surface free energy of explants $(\mathrm{p}=0.002)$.

Taken together, these data suggested that electron density was increased on skin surface after CS treatment, and reduced apolar interactions by lowering dispersive interactions [29].

\section{Biometrological probes and FT-IR spectroscopy}

CS-treated explants were then analyzed using biometrological probes and infrared spectroscopy in order to characterize skin barrier function modifications induced by cigarette smoke.

The mean $\mathrm{pH}$ value obtained for control explants was $5.42 \pm 0.74$, consistent with $\mathrm{pH}$ values in vivo [30]. The average $\mathrm{pH}$ obtained for CS-treated skin increased significantly and reached a value of $5.92 \pm 0.68$

In addition, the mean TEWL value measured for control explants was $15.5 \pm 4.8 \mathrm{~g} / \mathrm{h} / \mathrm{m}^{2}$ compared to a significant ( $\mathrm{p}<0.05$ ) increase to $21.4 \pm 6.9 \mathrm{~g} / \mathrm{h} / \mathrm{m}^{2}$ for CS treated explants.

Finally, infrared spectroscopic confirmed the CS impact on skin barrier function. We focused analyses on $\mathrm{CH}_{2}$ symmetric stretch wavelength $\left(v_{\mathrm{sym}} \mathrm{CH}_{2}\right)$. According to Mendelsohn et al. [31], the corresponding wavelength allows quantitative monitoring of chain conformation order and packing of lipid bilayers in the stratum corneum. As highlighted by temperature induced modifications, solid-solid transitions can be observed through the evolution of $v_{\text {sym }} \mathrm{CH}_{2}$. A first orthorhombic $\rightarrow$ hexagonal transition is observed at a wavelength around $2849.5 \mathrm{~cm}^{-1}$ followed by a hexagonal $\rightarrow$ disorder packing transition observed around 2852 $\mathrm{cm}^{-1}$ [31]. In the present study, as shown on fig. 2, the mean $v_{\mathrm{sym}} \mathrm{CH}_{2}$ observed for control explants was $2851.01 \pm 0.35 \mathrm{~cm}^{-1}$, corresponding to a hexagonal packing. Such packing is usually observed at the skin surface [31]. However, the mean $v_{\text {sym }} \mathrm{CH}_{2}$ observed for CS treated explants was significantly increased $\left(2851.43 \pm 0.46 \mathrm{~cm}^{-1}, \mathrm{p}<0.05\right)$. This change in 
wavelength is associated with more flexible chain of lipids and more disordered lipid chain packing.

\section{Impact of cigarette smoke on skin transcriptome and protein expression}

To investigate how the physico-chemical parameters reflect alterations of gene expression, transcription profiles were analyzed. An efficient stress activation in HSE after 1CS or 2CS exposure was indeed observed through the induction of AHR and NRF2 -target genes, as well as MMP1, MMP3 genes encoding for metalloproteinases (Table S2) as described in several studies $[13,16,17,22]$. In contrast to the AHR target genes (CYP1A1 and CYP1B1) for which induction levels were quite close following 1CS or 2CS exposure, the induction of the majority of NRF2 target genes was higher with smoke from 2 cigarettes. Similarly, the induction of the MMP1 and MMP3 genes confirmed that the stress generated is higher with 2 CS. However, pro-inflammatory genes were not significantly modulated in CS-exposed explants. In contrast to oxidative stress, it is possible that the absence of modulated proinflammatory genes cannot be detected at the chosen time window (24hrs after CS exposure). Thus, the following results are based on the 2CS exposed HSE from 4 volunteers. Noteworthy, our molecular analyses showed modulations of specific genes, which should cause mitochondrial dysfunction as well as an altered elimination of oxidized lipids as illustrated by the downregulation of genes such as NDUFA4L2, ATP1A1, ALDH7A1, ALDH2 and PRODH (Fig. 3 and Table S4). This observation was supported by the increase of MDA $(+37 \%, p<0.0001)$, a marker of lipid peroxidation, detected in the culture medium of CS-treated HSE (Fig. 4).

The NDUFA4L2 protein is located in the mitochondria and is considered to be a crucial mediator that regulates cell survival [32]. For instance, it has been shown that silencing of NDUFA4L2 (NADH dehydrogenase (ubiquinone) 1 alpha subcomplex 4-like 2) affects cell viability, increases mitochondrial mass, and induces ROS generation in hypoxia in various cancer cells (non-small cell lung cancer, renal cell carcinoma) [33]. ALDH2 (aldehyde dehydrogenase 2) is a mitochondrial enzyme metabolizing acetaldehyde and other toxic aldehydes, such as 4-hydroxynonenal (4-HNE). Inhibition of $A L D H 2$ by siRNA altered cell functions together with the impairment of bioenergetic functions and a decrease in mitochondrial respiration. Additionally, loss of ALDH2 reduced cell proliferation and migration and increased paracellular permeability [34]. Associated with a regulation of mitochondrial respiration, $P R O D H$ (proline dehydrogenase/oxidase), which encodes the first 
enzyme of the pathway of proline catabolism can be channeled to produce ATP for cell survival under low glucose conditions with or without concurrent hypoxia [35]. PRODH expression is often repressed in various tumors and in situations that limiting mitochondrial proline utilization. Proline availability for PRODH-dependent ATP or ROS generation depends on the activity of prolidase and on the use of proline during collagen biosynthesis [36]. Indeed, the dermal architecture should be likely altered in response to CS since we observed a repression of COL1A2, COL9A3 and LOX (lysyl oxidase) genes together with an induction of metalloproteinases genes $M M P 1$ and $M M P 3$. Recent works have shown that knockdown of ATP1A1, an $\alpha$-subunit of $\mathrm{Na}+\mathrm{K}+$ ATPase, induces mitochondrial dysfunction by disrupting ion homeostasis that results in apoptosis [37]. This $\alpha$ subunit has 10 transmembrane segments known as FXYD segments, with variable binding affinity for different regulators or modulators, particularly lipid molecules. $\mathrm{Na}+\mathrm{K}+$-ATPase activity is compromised by high production of ROS and in particular by lipid peroxidation products [38]. Lipid peroxidation and oxidative stress can be negatively regulated by induction of ANGPTL4 [39]. Its downregulation in our study might indicate that these processes are not negatively controlled at least $24 \mathrm{~h}$ after CS exposure. Similarly, bioactive oxidized lipids may be degraded by antioxidant enzymes; such as paraxonase 2 encoded by the PON2 gene [40], which is widely expressed in a variety of tissues, including intestinal epithelial cells, vascular endothelial cells, kidney, cardiomocytes, and keratinocytes. Overexpression of PON2 reduces oxidative status, prevents cell apoptosis and inhibits cell-mediated low-density lipoprotein oxidation. In contrast, PON2 knock-down caused vast reactive oxygen species formation and stimulated mitochondrial cell death signaling [41]. PON2 expression was decreased both at the gene and protein level (Fig. 3 and 4) under CS treatment supporting ongoing prooxidative activities. According to these observations at a transcriptional level, our results highlight the ability of CS to promote the production of ROS as a result of the decrease of mitochondrial activities in HSE. These findings were confirmed by immunohistochemistry, as revealed by the increase of PINK-1, a marker of damaged mitochondria, in the viable epidermis (Fig. 4). This mitochondrial alteration is responsible for changes in other processes such as lipid peroxidation, decreased collagen biosynthesis, and increased collagen degradation via the induction of metalloproteinases. In line with reported observations $[42,43]$, the induction of several NRF2 target genes (HMOX1, AKR1C1, AKR1C3, PRDX1, SLC7A11, SRXN1, TXNRD1) was detected in HSE exposed to CS. Among them, NRF2 and AKR1C3 protein expressions were also confirmed by immunostaining (Fig. 4). 
271 In addition, repression of $C A 9$ (carbonic anhydrase IX) and $A Q P 1$ (aquaporin 1) genes was 272 observed in our transcriptomic analysis. The cellsurface $\mathrm{pH}$ regulatory enzyme, CA9, 273 catalyzes the reversible hydration of carbon dioxide to acidify the cellular microenvironment 274 and also provides an extracellular $\mathrm{pH}$ control mechanism [44]. In normal skin, the highest 275 expression of $C A 9$, whose inhibition can contribute to increase cellular $\mathrm{pH}$, was detected in 276 hair follicles, sebaceous glands, and basal parts of epidermis [45]. AQP1 expression which 277 was found to be increased with $\mathrm{pH}$ [46], is correlated with the regulation of epidermal 278 hydration and might influence skin water transport [47].

279

280 
283

284

285

286

287

288

289

290

291

292

293

294

295

296

297

298

299

300

301

302

303

304

305

306

307

308

309

310

311

312

313

314

The observed alteration of the skin surface physico-chemistry and the skin surface free energy can result from a modification of the lipid composition as highlighted by Mavon et al. [24]. Especially, the basic component $\gamma^{-}$results from the amount of amphiphilic lipids, namely the free fatty acids (FFAs), present in the skin surface because their carbonyl groups enriched in electrons induce basic interactions.

This observation can also be explained by the oxidation of surface lipids caused by CS exposure $[48,49]$. Indeed, it has been shown that CS triggers an oxidative stress favoring the peroxidation of skin lipids thus reducing its anti-oxidant content. This oxidation leads to the formation of new oxygenated chemical species and amphiphilic molecules such as epoxides, peroxides or carbonyls [50].

The chemical composition of such oxidized compounds, their possible chemical structures and the mechanisms of their formation were deeply described in the study of Eudier et al. [28] in the case of squalene oxidation. As a consequence, the corresponding compounds, enriched in electrons, are able to establish additional hydrogen bonds thus increasing interactions with polar liquids such as water, as illustrated by contact angle measurements on CS treated explants (Fig. 1). It is well established that those compounds are responsible for the increase of basic interactions [24] and the decrease of dispersive interactions [29].

By modifying skin surface physico-chemistry, CS treatment alters the way the skin interacts with its environment. A significant increase of skin wettability can impact skin barrier function and skin interactions with topic products [24].

The rise of $\mathrm{pH}(\mathrm{p}<0.0001)$ was consistent with the physico-chemical data indicating an enhanced basic behavior of treated skin surface. These observations confirmed the suggested influence of CS on skin permeability. Indeed, skin $\mathrm{pH}$ can influence the diffusion of drugs and their distribution in skin layers [30].

Parallel with the $\mathrm{pH}$ increase, the mean TEWL increases also significantly after CS treatment. According to Elkeeb et al. [51], the TEWL measurement is an accurate in vitro approach to evaluate skin water barrier function. An increase in TEWL following CS exposure indicates also an alteration of the skin barrier.

Finally, FTIR results demonstrated a lipid chain packing disorder. This chain packing reflects skin barrier function integrity [50]. Highly ordered lipids provide impermeability needed for the water barrier function whereas disordered lipids bilayers may increase skin permeability [31]. Consequently, skin barrier function was disrupted in CS treated explants, consistent with 
the TEWL results [51,52] as proposed on fig. S1. Such a disorganization of lipids chain bilayers can be explained by the formation of lipid oxidation compounds, perturbing the original lipid bilayer organization [53].

Predictable, genes involved in the AHR-pathway were induced in CS-exposed HSE, our molecular analysis suggested that mitochondrial functions were strongly impacted and oxidized lipids failed to be eliminated promoting skin barrier alteration. Indeed, the various environmental toxicants are known to induce the AhR translocation to the nucleus and interact with AhR nuclear translocator (ARNT) and binds to the Xenobiotic Response Element DNA domain (XRE). Once the complex is bound, it initiates the transcription of genes such as CYP1A1, CYP1B1, which are involved in cell detoxification [54]. However, the AhR-dependent protection could not shield cells from constant oxidative stress [55] and prolonged ROS production by the repression of several key enzymes (i.e NDUFA4L2 and PRODH), as illustrated in our study (Fig. 3), would indeed enhance oxidative damage to mitochondria and in fine the elimination of oxidized lipids might be impacted. Downregulation of ATP1A1 (repressed in our study), whose activity is compromised in response to a high generation of ROS or by lipid peroxidation products, induces also mitochondrial dysfunction by disrupting iron homeostasis. The presence of lipid peroxidation products was also confirmed by an increase in MDA of $37 \%(\mathrm{p}<0.0001)$. Furthermore, oxidized lipids can be degraded by paraxonase 2 encoded.

Overexpression of this gene reduces the oxidative status and inhibits cell-mediated lowdensity lipoprotein oxidation. Since expression of this gene is repressed, it can be expected that its activity should also decrease leading to LDL/HDL oxidation and mitochondrial apoptosis/oxidation (see Fig. 3). Therefore, according to these findings, besides an oxidative stress favoring the peroxidation of skin lipids and triggered by the modulation of these genes, it can be assumed that the activities of the encoded protein will produce in turn new oxygenated chemical species and amphilic molecules such as expoxides, peroxides, or carbonyls.

342 Exposure of mitochondria to cellular stress alters their fusion/fission dynamics and promotes excessive ROS production, an accelerator of cellular senescence. Whereas mild stress induces a prosurvival response termed stress-induced mitochondrial hyperfusion, severe stress results in mitochondrial fragmentation and mitophagy [56]. In the present study, the mitophagy was illustrated through the induction of HMOXI and confirmed by immunostaining of LC3B and PINK1 (Fig. 4). In response to the mitochondrial dysfunction, induction of NRF2-regulated 
genes was not surprising since such an induction is an indicator of the activation of a cellular protection against the pro-oxidative process and the need to neutralize ROS in the outer layers of the epidermis.

Polyunsaturated fatty acids in cells and organelles membranes are primary targets for ROS attack, which may lead to lipid peroxidation and generation of reactive lipids. Indeed, the presence of lipids such as 4-hydroxynonenal was supported by an increase in MDA of 37\% $(\mathrm{p}<0.0001)$.

355 Thus, the CS-induction of lipid oxidation is detected by the physico-chemistry approach, results in an oxidative stress due to the modulation of genes expression.

Moreover, the repression of genes such as $C A 9$ and $A Q P 1$ provided some clues to explain the alterations of the skin barrier after CS exposure through an increase of the cellular $\mathrm{pH}$ [45] and the altered water transport [47], respectively. Although the major intake of tobacco smoke toxicants is through the inhalation of secondhand smoke, our results show that the accumulation of toxicants in the skin is equally relevant as shown by ex vivo CS exposure. Epidermal absorption is usually overlooked as a possible pathway of exposure, but it is especially pertinent in the case of environment pollution where nonsmokers are exposed to smoke toxicants. As observed by Weschler and Nazaroff, many semi-volatile organic compounds like nicotine can be dermally absorbed and transported to the dermal blood supply [57]. Despite the absence of an inflammatory response that may be also transient compared to the conditions of active smoking, this is the first time that an ex vivo study demonstrates alterations in response to smoking, probably closer to the physiological conditions of passive smoking which may contribute to skin aging.

371 In the future, it would be interesting to evaluate with this ex vivo model the effects on the skin 372 of e-cigarettes via the Pollubox ${ }^{\circledR}$ device and to study the impact of cigarette smoke extracts on human skin explants ex vivo following systemic application (in the culture medium) in order to mimic more closely active smoking environment. 


\section{Acknowledgements}

377 This study has been led within the framework of the URBASKIN project, selected as Project 378 of Excellence by the General Directorate for Competitiveness Industry and Services (DGCIS) 379 in the 20th Appeal FUI (Fonds Unique Interministériel). It has been financed by BPI France 380 and by Normandie, Val de Loire and Ile-de-France regions and supported by the Cosmetic 381 Valley. The authors sincerely thank contributors and funders for their support.

382 We also thank Caroline Durand and Marie Reynier for HSE preparation and treatment and 383 Cynthia Fays, Tatiana Judith, Laurent Peno-Mazzarino, Ulduz Faradova and Stéphanie 384 Scalvino for their support in skin sample processing for microscopy observation and data 385 analysis.

386 We thank, Laurine Martinelli and Tessa Mitric for help during their training and Claudine 387 Deloménie and Florent Dumont from the UMS IPSIT (Institut Paris Saclay Innovation 388 Thérapeutique) platform for their collaboration.

\section{Author contributions}

390 GP, AP, FE, CP, GS and PB wrote the main manuscript.

GP, EL, AP, FE, GS and CP developed the study design.

AP, TB and PB performed the genomic experiments.

GP was in charge to carry out the microscope analysis.

FE, MG, GS and CP realized the physical-chemical characterization of human skin surface.

All the authors took part in the scientific discussions of the obtained results and have read and approved the manuscript.

The study was conducted in accordance with the ethical policies of Experimental Dermatology. The Bio-EC Laboratory possesses an authorization from the French Minister for Health.

\section{Conflict of interest}

The authors have declared no conflicting interests. 


\section{REFERENCES}

406

407

[1] C.P. Wild, Complementing the genome with an exposome: the outstanding challenge of 408 environmental exposure measurement in molecular epidemiology, Cancer Epidemiol. Biomarkers Prev. 2005, 14, 1847-1850.

410 [2] J. Krutmann, A. Bouloc, G. Sore, B.A. Bernard B, T. Passeron, The skin aging exposome, 411 Dermatol. Sci. 2017, 85, 152-161.

412 [3] IARC Working Group on the Evaluation of Carcinogenic Risks to Humans. Tobacco 413 smoke and involuntary smoking. IARC Monogr Eval Carcinog Risks Hum. 2004, 83, 1-1438.

414 [4] R. Talhout, T. Schulz, E. Florek, J. van Benthem, P. Wester, A. Opperhuizen, Hazardous 415 compounds in tobacco smoke. Int. J. Environ Res. Public Health. 2011, 8, 613-628.

416 [5] J. Ticha, C. Wright, Rapid detection of toxic compounds in tobacco smoke condensates 417 using high-resolution ${ }^{1} \mathrm{H}$ nuclear magnetic resonance spectroscopy. Anal. Methods. 2016, 8, $418 \quad 6388-6397$.

[6] HHS (Department of Health and Human Services). How tobacco smoke causes disease: The biology and behavioral basis for smoking-attributable disease: A report of the Surgeon 421 General. Office on Smoking and Health; Atlanta, GA 2010.

422 [7] A. Thiele., H. Klus, L. Müller, Tobacco smoke: Unraveling a controversial subject. Exp. 423 Toxicol. Pathol. 2008, 60, 141-156.

424 [8] D. G Smith, Effect of passive smoking on health, BMJ. 2003, 326, 1048-1049.

[9] M. Oberg, M. S. Jaakkola, A. Woodward, A. Peruga, A. Pruss-Ustun, Worldwide burden countries. Lancet. 2011, 377, 139-146.

[10] H. W. Daniell. Smoker's wrinkles study in the epidemiology of crow's feet. Ann intern med. 1971, 75, 873-880.

[11] D. N. Doshi, K. K. Hanneman, K. D. Cooper. Smoking and skin aging in identical twins. Arch Dermatol. 2007, 143, 1543-1546.

[12] H. C. Okada, B. Alleyne, K. Varghai, K. Kinder, B. Guyuron. Facial changes caused by smoking: a comparison between smoking and non-smoking identical twins. Plas. Rec. Surg. 2013, 132, 1085-1092.

[13] C. Lahmann, J. Bergemann, G. Harrison, A. R. Young. Matrix metalloprotease-1 and 436 skin ageing in smokers. Lancet, 2001, 357, 935-936. 
[14] H. Tanaka, Y. Ono, S. Nakata, Y. Shintani, N. Sakakibara, A. Morita, Tobacco smoke extract induces premature skin aging in mouse skin. J Dermatol Sci, 2001, 46, 69-71.

[15] G. Y. Yang, C. L. Zhang, X. C. Liu, G. Qian, D. Q. Deng. Effects of cigarette smoke extracts on the growth and senescence of skin fibroblasts in vitro. Int. J. Biol. Sci. 2013, 9, $613-623$.

[16] Y. Ono Y, K. Torii, E. Fritsche, Y. Shintani, E. Nishida, M. Nakamura, Y. Shirakata, T. Haarmann-Stemmann, J. Abel, J. Krutmann, A. Morita, Role of the aryl hydrocarbon receptor in tobacco smoke extract-induced matrix metalloproteinase-1 expression. Exp. Dermatol. 2013, 22, 349-353.

[17] M. Nakamura, Y. Ueda, M. Hayashi, H. Kato, T. Furuhashi, A. Morita. Tobacco smokeinduced skin pigmentation is mediated by the aryl hydrocarbon Receptor. Exp. Dermatol., 2013, 22, 556-558.

[18] C.H. Lee CH, H. Y. Chuang, C. H. Hong, S. K. Huang, Y. C. Chang, Y. C. Ko, H. S. Yu, Lifetime exposure to cigarette smoking and the development of adult-onset atopic dermatitis. Br. J. Dermatol. 2011, 164, 483-489.

[19] M. Sopori. Effects of cigarette smoke on the immune system. Nat. Rev. Immunol. 2002, $2,372-377$.

[20] L. S. Wong, H. M. Green, J. E. Feugate, M. Yadav, E.A. Nothnagel, M. Martins-Green. Effects of "second-hand" smoke on structure and function of fibroblasts, cells that are critical for tissue repair and remodelling. BMC. Cell. Biol. 2004, 5, 13.

[21] D. Thorne, J. Adamson, A review of in vitro cigarette smoke exposure systems. Exp. Toxicol Pathol. 2013, 65, 1183-1193.

[22] S. Lecas, E. Boursier, R. Fitoussi, K. Vié, I. Momas, N. Seta, S. Achard. In vitro model adapted to the study of skin ageing induced by air pollution. Toxicol Lett. 2016, 259, 60-68.

[23] F. Eudier, G. Savary, M. Grisel, C. Picard. Skin surface physico-chemistry: Characteristics, methods of measurement, influencing factors and future developments. $A d v$ Colloid Interface Sci. 2019, 264, 11-27.

[24] A. Mavon, H. Zahouani, D. Redoules, P. Agache, Y. Gall, P. Humbert. Sebum and stratum corneum lipids increase human skin surface free energy as determined from contact angle measurements: a study on two anatomical sites. Colloids Surf. B Biointerfaces. 1997, 8, 147-155.

[25] G. Percoco, C. Merle, T. Jaouen, Y. Ramdani, M. Bénard, M. Hillion, L. Mijouin, E. Lati, M. Feuilloley, L. Lefeuvre, A. Driouich, M.L. Follet-Gueye. Antimicrobial peptides and 
pro-inflammatory cytokines are differentially regulated across epidermal layers following bacterial stimuli. Exp. Dermatol. 2013, 22, 800-806. [26] C. J. Van Oss, R. J. Good, M. K. Chaudhury, Additive and Nonadditive Surface Tension 285-293.

[29] C. J. Van Oss, M. K. Chaudhury, R. J. Good, Monopolar surfaces. Adv. Colloid Interface

[30] H. Wagner, K.-H. Kostka, C.-M. Lehr, U.F. Schaefer. pH profiles in human skin:

482

483 influence of two in vitro test systems for drug delivery testing. Eur. J. Pharm. Biopharm. $\mathbf{2 0 0 3}, 55,57-65$.

[31] R. Mendelsohn, C.R. Flach, D.J. Moore. Determination of molecular conformation and permeation in skin via IR spectroscopy, microscopy, and imaging. Biochim. Biophys. Acta, Biomembr. 2006, 1758, 923-933.

[32] L. Meng, X. Yang, X. Xie, M. Wang. Mitochondrial NDUFA4L2 protein promotes the vitality of lung cancer cells by repressing oxidative stress. Thorac Cancer. 2019, 10, 676-685. [33] G. Lucarelli, M.Rutigliano, F. Sallustio, D. Ribatti, A. Giglio, M. Lepore Signorile, V. Grossi. Integrated multi-omics characterization reveals a distinctive metabolic signature and the role of NDUFA4L2 in promoting angiogenesis, chemoresistance, and mitochondrial dysfunction in clear cell renal cell carcinoma. Aging, 2018, 10, 3957-3985.

[34] G. Nannelli, E. Terzuoli, V. Giorgio, S. Donnini, P. Lupetti, A. Giachetti, P. Bernardi, M. Ziche. ALDH2 Activity Reduces Mitochondrial Oxygen Reserve Capacity in Endothelial Cells and Induces Senescence Properties. Oxid Med Cell Longev. 2018, 9765027.

[35] W. Liu, JM. Phang. Proline dehydrogenase (oxidase), a mitochondrial tumor suppressor, and autophagy under the hypoxia microenvironment. Autophagy. 2012, 8, 1407-1409.

[36] I. Zareba, J.Palka. Prolidase-proline dehydrogenase/proline oxidase-collagen biosynthesis axis as a potential interface of apoptosis/autophagy. Biofactors. 2016, 42, 341348

[37] S. Takase, R. Kurokawa, D. Arai, K. Kanto Kanemoto, T. Okino, Y.Nakao, T. Kushiro, M. Yoshida, K. Mastumoto. A quantitative shRNA screen identifies ATP1A1 as a gene that regulates cytotoxicity by aurilide B. Sci Rep. 2017, 7, 2002. 
504

505

506

507

508

509

510

511

512

513

514

515

516

517

518

519

520

521

522

523

524

525

526

527

528

529

530

531

532

533

534

535

536

[38] S. Roy, A. Dasgupta, U. Banerjee, P. Chowdhury, A. Mukhopadhyay, G. Saha, O. Singh. Role of membrane cholesterol and lipid peroxidation in regulating the $\mathrm{Na}+\mathrm{K}+$-ATPase activity in schizophrenia. Indian J Psychiatry. 2016, 58, 317-325.

[39] A. Georgiadi, L. Lichtenstein, T. Degenhardt, MV. Boekschoten, M. van Bilsen, B. Desvergne, M. Müller, S. Kersten. Induction of cardiac Angptl4 by dietary fatty acids is mediated by peroxisome proliferator-activated receptor beta/delta and protects against fatty acid-induced oxidative stress. Circ Res. 2010, 106, 1712-1721.

[40] J. Marsillach, J. Camps, R. Beltran-Debón, A. Rull, G. Aragones, C. Maestre-Martínez, F. Sabench. Immunohistochemical analysis of paraoxonases-1 and 3 in human atheromatous plaques. Eur J Clin Invest. 2011, 41, 308-314.

[41] I. Witte, S. Altenhöfer, P. Wilgenbus, J. Amort, AM. Clement, A. Pautz, H. Li, U. Förstermann, S. Horke. Beyond reduction of atherosclerosis: PON2 provides apoptosis resistance and stabilizes tumor cells. Cell Death Dis. 2011, 13, 2:e112.

[42] WP. Vermeij, A. Alia, C. Backendorf. ROS quenching potential of the epidermal cornified cell envelope. J. Invest. Dermatol. 2011, 131, 1435-1341.

[43] P. Quantin, A. Patatian, M. Floreani, C. Egles, P. Benech, H. Ficheux. Temporal transcriptomic analysis of human primary keratinocytes exposed to $\beta$-naphthoflavone highlights the protective efficacy of skin to environmental pollutants. Toxicol In Vitro. 2020, $65,104822$.

[44] S.H. Lee, D. McIntyre, D. Honess, A. Hulikova, J. Pacheco-Torres, S. Cerdán, P. Swietach, A.L. Harris, J.R. Griffiths. Carbonic anhydrase IX is a $\mathrm{pH}$-stat that sets an acidic tumour extracellular pH in vivo. Br J Cancer. 2018, 119, 622-630.

[45] L. Syrjänen, T. Luukkaala, M. Leppilampi, M. Kallioinen, S. Pastorekova, J. Pastorek, A. Waheed, WS. Sly, S. Parkkila, T. Karttunen. Expression of cancer-related carbonic anhydrases IX and XII in normal skin and skin neoplasms. APMIS, 2014, 122, 880-889.

[46] Y. Zhai, H. Xu, Q. Shen, F. Schaefer, CP. Schmitt, J. Chen, H. Liu, J. Liu, J. Liu. pHmediated upregulation of AQP1 gene expression through the Spi-B transcription factor. BMC Mol Biol. 2018, 19, 4.

[47] J. Agren, S. Zelenin, M. Håkansson, A.C. Eklöf, A. Aperia, LN. Nejsum, S. Nielsen, G. Sedin. Transepidermal water loss in developing rats: role of aquaporins in the immature skin. Pediatr Res. 2003, 53, 558-565.

[48] M. Egawa, Y. Kohno, Y. Kumano. Oxidative Effects of Cigarette Smoke on the Human Skin. Int. J. Cosmet. Sci. 1999, 21, 83-98. 
[49] B. Frei, T.M. Forte, B.N. Ames, C.E. Cross, Gas phase oxidants of cigarette smoke induce lipid peroxidation and changes in lipoprotein properties in human blood plasma. Protective effects of ascorbic acid. Biochem. J. 1991, 277, 133-138.

[50] K.M. Schaich, Thinking outside the classical chain reaction box of lipid oxidation. Lipid Technol. 2012, 24, 55-58.

[51] R. Elkeeb, X. Hui, H. Chan, L. Tian, H.I. Maibach. Correlation of transepidermal water loss with skin barrier properties in vitro: comparison of three evaporimeters. Skin Res. Technol. 2010, 16, 9-15.

[51] M. Denda, J. Koyama, R. Namba, I. Horii. Stratum corneum lipid morphology and transepidermal water loss in normal skin and surfactant-induced scaly skin. Arch. Dermatol. 1994, 286, 41-46.

[52] B. Forslind. A domain mosaic model of the skin barrier. Acta Derm. Venereol. 1994, 74, $1-6$.

[53] D. Borchman, O.P. Lamba, S. Salmassi, M. Lou, M. Cecilia Yappert. The dual effect of oxidation on lipid bilayer structure. Lipids. 1992, 27, 261-265.

[54] H. Uchi, M. Yasumatsu, S. Morino-Koga, C. Mitoma, M. Furue. Inhibition of aryl hydrocarbon receptor signaling and induction of NRF2-mediated antioxidant activity by cinnamaldehyde in human keratinocytes. J Dermatol Sci. 2017, 85, 36-43.

[55] Y.H. Cheng, SC. Huang, C.J. Lin, L.C. Cheng, L.A. Li. Aryl hydrocarbon receptor protects lung adenocarcinoma cells against cigarette sidestream smoke particulates-induced oxidative stress. Toxicol Appl Pharmacol. 2012, 259, 293-301.

[56] M. Frank, S. Duvezin-Caubet, S. Koob, A. Occhipinti, R. Jagasia, A. Petcherski, M.O. Ruonala, M. Priault, B. Salin, A.S. Reichert. Mitophagy is triggered by mild oxidative stress in a mitochondrial fission dependent manner. Biochim Biophys Acta. 2012, 1823, 2297-2310. [57] C. J. Weschler, W. W. Nazaroff, Dermal Uptake of Organic Vapors Commonly Found in Indoor Air Environ Sci Technol. 2014, 48, 1230-1237. 
Figure 1: (a) Advancing contact angle measured on control and CS treated explants with water, diiodomethane and formamide and (b) surface free energy parameters calculated according to Van Oss model. $\gamma^{\mathrm{LW}}$ is the Lifshitz-van der Waals (apolar) component of the surface free energy of the liquid, $\gamma^{-}$and $\gamma^{+}$are the electron-donor and electron-acceptor (polar) parameters of the acid-base components of the liquid surface free energy, respectively.

572 Figure 2: Mean FTIR Spectra of control and CS-treated living skin explant with a zoom of 573 the lipid chain packing area.

Figure 3: Schematic representation of functional networks deduced from PredictSearch ${ }^{\circledR}$ analysis of the set of genes modulated in skin explants $24 \mathrm{~h}$ after exposure to CS. The legend of this figure is indicated at the bottom left corner. Induced genes are noted in blue and repressed genes in red.

578 Figure 4: Microscopical analysis and MDA dosage of skin explants upon CS exposure.

579 Abbreviations: SC, stratum corneum; E, epidermis; PD, papillary dermis.

580 Scale bar: $50 \mu \mathrm{m}$.

581 Figure S1: Proposed explanation for TEWL increase and skin barrier degradation in CS 582 treated explants.

583 Table S1: List of the different skin donors used in the present work.

Table S2: List of key genes evaluated in response to 1CS and 2 CS to determine dose effect on the stress stimulation on human skin explants

Table S3: List of the primary antibodies used in the present work. Abbreviations: HIER (Heat Induced Epitope Retrieval).

Table S4: List of modulated genes in response to CS2 and correlated in functional networks. Fold change (FC) were calculated from the mean values of 3 explants per condition (CS2vsT) generated from each donor (V1, V2, V3 and V4), $\mathrm{p}<0.05$ 